\title{
Classical and Bayesian Estimation of Stress-Strength Reliability from Generalized Inverted Exponential Distribution based on Upper Records
}

\author{
M.J.S. Khan \\ Department of Statistics and Operations Research \\ Aligarh Muslim University, India \\ Jahangirskhan@gmail.com \\ Bushra Khatoon \\ Department of Statistics and Operations Research \\ Aligarh Muslim University, India \\ bushra.stats@gmail.com
}

\begin{abstract}
This paper deals with the problem of classical and Bayesian estimation of stress-strength reliability from a generalized inverted exponential distribution (GIED) based on upper record values. Hassan et al. (2018) have discussed the maximum likelihood estimator (MLE) and Bayes estimator of $\mathrm{R}$ by considering that the scale parameter of defined distribution is known while we have considered the case when all the parameters of GIED are unknown. In classical approach, we have obtained MLE and uniformly minimum variance estimator (UMVUE). In Bayesian approach, we have considered the Bayes estimator of R by considering the squared error loss function. Further, based on upper records, we have considered the asymptotic confidence interval (CI) based on MLE, Bayesian credible interval and bootstrap CI for R. Moreover, to evaluate the performances of the discussed estimators of $\mathrm{R}$, a Monte Carlo simulation and a real data application have been carried out.
\end{abstract}

Keywords: Generalized inverted exponential distribution; stress-strength reliability; uniformly minimum variance unbiased estimator; Bayes estimator; confidence interval; upper record values.

AMS Classification: 62F10; 62F15; 62N05

\section{Introduction}

Let $X$ and $Y$ be two independent random variables and $R=P(X<Y)$ represents the probability that $X$ does not exceeds $Y$. In terms of stress-strength reliability, $X$ denotes the stress applied to a device having strength $Y$. For proper functionning of the device, the strength of the device $Y$ must exceeds to stress $X$. Stress-strength reliability model appears in many practical situations like, in medical sciences, structural engineering, natural phenomena like a flood, earthquakes, etc. In medical science, let $X$ and $Y$ stand for the effect of the control treatment and the new treatment respectively. When new treatment applied over a control treatment then the quantity $R=P(X<Y)$ express the effectiveness of the new treatment compared with the control treatment. In structural engineering, while building a bridge, $X$ and $Y$ shows the stress (load on the bridge) and strength (capacity) of the bridge respectively. Bridge survive only if the strength of the bridge is greater than the stress applied on it. In such a situation $R=P(X<Y)$ shows the survival probability of the bridge. In statistical literature, estimation of $R=P(X<Y)$ has been widely studied under the assumption that $X$ and $Y$ are independent random variables belonging to the same family of distributions. Several researchers have deduced the estimators of R. Awad et al. (1981) considered the MLE of $P(Y<X)$ when $X$ and $Y$ have 
a bivariate exponential distribution. For an extensive and lucid literature review regarding estimation and application of the stress-strength reliability, readers are referred to Johnson (1988) and Kotz et al. (2003).

In stress-strength reliability, the problem of estimating $R$ is carried out for different data sets such as complete, censored and so on. However, many situations are appeared in practical life where observations are more extreme than the current extreme values. A natural example is industrial stress testing where only items are destroyed which are more weaker than other observed failed items, see Ahmadi and Arghami (2003 a, b). This type of data is called "Record Data" or "Record Values". Chandler (1952) developed the mathematical theory of record values and discussed its basic properties. Consequently, many researchers considered record data for their work of interest. A detailed treatment with extensive references are provided by Ahsanullah (1995) and Arnold et al. (1998). Let $X_{1}, X_{2}, \ldots$ be an infinite sequence of identically and independently distributed (iid) random variables. An observation $X_{j}$ is called an upper record if $X_{j}>X_{i}$ for every $i<j$. We shall assume that $X_{j}$ occurs at time $j$, then the record time sequence is defined as $U_{1}=1$ and $U_{n}=\min \left\{j: X_{j}>X_{U_{n-1}}\right\}$. The upper record sequence $R_{1}, R_{2}, \ldots, R_{n}$ is defined as $R_{n}=X_{U_{n}}, n \in \mathrm{N}$. The joint probability density function ( $p d f$ ) of first $n$ upper records is given by

$$
f_{R_{1}, R_{2}, \ldots, R_{n}}\left(r_{1}, r_{2}, \ldots, r_{n}\right)=f\left(r_{n}\right) \prod_{i=1}^{n-1} \frac{f\left(r_{i}\right)}{\bar{F}\left(r_{i}\right)}, \quad r_{1}<r_{2}<\ldots<r_{n}
$$

where $\bar{F}\left(r_{i}\right)=1-F\left(r_{i}\right)$.

The exponential distribution played a very important role in reliability theory. Several researchers have considered its generalized and inverse form namely generalized exponential distribution (Gupta and Kundu, 1999) and inverted exponential distribution (Dey, 2007). Abouammoh and Alshingiti (2009) introduced the GIED in reliability estimation. Ghitany et al. (2013) discussed the likelihood estimation for a general class of inverted exponential distribution based on complete and censored samples. Further, several researchers considered the estimation of the parameters of GIED based on complete, censored samples and record values. For example: Dey and Dey (2014 a, 2014 b), Dey and Pradhan (2014), Dube et al. (2016), Dey et al. (2016), Panahi (2017) and Gunasekera (2018) and so on. The cumulative distribution function $(c d f)$ of $\operatorname{GIED}(\lambda, \alpha)$ is written as

$$
F(t)=1-\left[1-\exp \left(\frac{-\lambda}{t}\right)\right]^{\alpha}, \quad t \geq 0, \alpha, \lambda>0
$$

and its $p d f$ is given by-

$$
f(t)=\left(\frac{\alpha \lambda}{t^{2}}\right) \exp \left(\frac{-\lambda}{t}\right)\left[1-\exp \left(\frac{-\lambda}{t}\right)\right]^{\alpha-1}, \quad t \geq 0, \alpha, \lambda>0
$$

Estimation of parameters based on record values with different lifetime models have been discussed by various researchers. However, estimation of stress-strength reliability based on record values has got more attention in last two decades. Baklizi (2008 a, 2014 a) has considered the MLE, associated CIs and Bayesian inference of stress-strength reliability using record values for the exponential distribution. Interval estimation (Bayesian 
interval, bootstrap interval and interval using the generalized pivot variable) of the stressstrength reliability in two-parameter exponential distribution based on upper records has been obtained by Baklizi (2014 b). Baklizi (2008 b) and Wong and Wu (2009) have discussed the MLE, Bayesian estimation and interval estimation of $P(X<Y)$ respectively using lower record values from the generalized exponential distribution. Hassan et al. (2015) have described the estimation of stress-strength reliability for exponentiated inverted weibull distribution based on lower record values. Basirat et al. (2016) have derived the estimation of stress strength parameter for proportional hazard rate models for upper record values. Condino et al. (2018) have considered a similar problem for proportional reversed hazard model based on lower records. Khan and Arshad (2016) have studied the UMVU estimation of reliability function and stressstrength reliability from proportional reverse hazard family based on lower records. MLE, approximate Bayes estimator and the exact CIs of stress-strength reliability for the two-parameter bathtub-shaped lifetime distribution based on upper record values have been deduced by Tarvirdizade and Ahmadpour (2016). Mahmoud et al. (2016) have deduced the result for the Bayesian estimation of $P(X<Y)$ for the Lomax distribution based on upper record values. In this paper, Mahmoud et al. (2016) described the MLE of stress-strength reliability in two cases, when all the parameters are unknown and when scale parameter is common and known. Amin (2017) has discussed the estimation of stress-strength reliability based on upper record values for Kumaraswamy Exponential distribution. Recently, Dhanya and Jeevavand (2018) have considered the Bayesian estimation of squared error loss function and linex loss function and MLE of stressstrength reliability for power function distribution with different shape and same scale parameter based on records. Inference for the two-parameter bathtub-shaped distribution based on record data has been considered by Raqab et al. (2018). Rasethuntsa and Nadar (2018) have discussed the MLE, its asymptotic distribution and Bayes estimator under symmetric squared error loss function of stress-strength reliability in a multi-component system with nonidentical component strengths from a family of Kumaraswamy generalized distribution based on upper records. Khan and Khatoon (2019) have obtained MLE, UMVUE and Bayesian estimaor of stress strength reliability for exponential distribution based on generalized order statistics. In this paper, we have derived the classical (MLE and UMVUE) and Bayesian estimators of stress-strength reliability based on upper record values from GIED by taking common scale parameter and different shape parameter.

The rest of the paper is organized as follows: In Section 2, the MLE of $R=P(X<Y)$ is computed. Section 3 provided the asymptotic confidence interval and percentile bootstrap interval of stress-strength reliability. UMVUE and Bayesian inference of $R=P(X<Y)$ are discussed in Section 4 and 5 respectively. In Section 6, Monte Carlo simulations are carried out to check the efficiency of aforesaid estimators of R. A real data example is presented in Section 7 for the purpose of illustration.

\section{Maximum Likelihood Estimation}

In this Section, we consider the problem of estimating $R=P(X<Y)$ based on upper record values from GIED. Here, we obtained MLE of $\mathrm{R}$ by assuming that all the parameters of GIED are unknown. 
Let $\mathrm{X} \sim \operatorname{GIED}(\lambda, \alpha)$ and $\mathrm{Y} \sim \operatorname{GIED}(\lambda, \beta)$ be independent random variables. Let $R=P(X<Y)$ be the stress-strength reliability and it can be seen that $R=\frac{\alpha}{\alpha+\beta}$. We are interested to obtain the MLE of $R$ based on upper records. However, to find the MLE of $R$, it is required to obtain the MLE of $\alpha$ and $\beta$ say, $\hat{\alpha}_{M L}$ and $\hat{\beta}_{M L}$. By using invariance property of MLE, one can find the MLE of $R$. Let $X_{1}, X_{2}, \ldots$ be a sequence of iid random variables having the parent population $\operatorname{GIED}(\lambda, \alpha)$ and $\underline{r}=r_{1}, r_{2}, \ldots, r_{n}$ be the corresponding set of first $n$ upper records. Similarly, let $Y_{1}, Y_{2}, \ldots$ be another sequence of iid random variables having the parent population $\operatorname{GIED}(\lambda, \beta)$ and the corresponding set of first $m$ upper records are $s=s_{1}, s_{2}, \ldots, s_{m}$. Then the likelihood function based on these upper records values is given by

$$
L(\alpha, \beta, \lambda \mid \underline{r}, \underline{s})=\left(f\left(r_{n}\right) \prod_{i=1}^{n-1} \frac{f\left(r_{i}\right)}{\bar{F}\left(r_{i}\right)}\right)\left(g\left(s_{m}\right) \prod_{i=1}^{m-1} \frac{g\left(s_{i}\right)}{\bar{G}\left(s_{i}\right)}\right), r_{1}<r_{2}<\ldots<r_{n},
$$

$$
s_{1}<s_{2}<\ldots<s_{n}
$$

where $f, F$ and $g, G$ represent the $p d f$, $c d f$ of $\operatorname{GIED}(\lambda, \alpha)$ and $\operatorname{GIED}(\lambda, \beta)$ respectively. Also $\bar{F}\left(r_{i}\right)=1-F\left(r_{i}\right)$ and $\bar{G}\left(s_{i}\right)=1-G\left(s_{i}\right)$. Putting the values of $f, F, g$ and $G$ in (2.1), the likelihood function can be written as

$$
L(\alpha, \beta, \lambda \mid \underline{r}, \underline{s})=\alpha^{n} \beta^{m} \lambda^{n+m}\left(1-e^{-\lambda / r_{n}}\right)^{\alpha}\left(1-e^{-\lambda / s_{m}}\right)^{\beta} \prod_{i=1}^{n} \frac{e^{-\lambda / r_{i}}}{r_{i}^{2}\left(1-e^{-\lambda / r_{i}}\right)} \prod_{i=1}^{m} \frac{e^{-\lambda / s_{i}}}{s_{i}^{2}\left(1-e^{-\lambda / s_{i}}\right)}
$$

Thus the log-likelihood function of the above expression is given by

$$
\begin{aligned}
l(\alpha, \beta, \lambda \mid \underline{r}, \underline{s})= & n \ln \alpha+m \ln \beta+(n+m) \ln \lambda+\alpha \ln \left[1-e^{-\lambda / r_{n}}\right]+\beta \ln \left[1-e^{-\lambda / s_{m}}\right]-\sum_{i=1}^{n} \frac{\lambda}{r_{i}} \\
& -2 \sum_{i=1}^{n} \ln r_{i}-\sum_{i=1}^{n} \ln \left[1-e^{-\lambda / r_{i}}\right]-\sum_{i=1}^{m} \frac{\lambda}{s_{i}}-2 \sum_{i=1}^{m} \ln s_{i}-\sum_{i=1}^{m} \ln \left[1-e^{-\lambda / s_{i}}\right]
\end{aligned}
$$

where, $l(\alpha, \beta, \lambda \mid \underline{r}, \underline{s})=\ln L(\alpha, \beta, \lambda \mid \underline{r}, \underline{s})$.

The MLE of $\alpha, \beta$ and $\lambda$ can be obtained by solving $\frac{\partial l(\alpha, \beta, \lambda \mid \underline{r}, \underline{s})}{\partial \alpha}=0, \frac{\partial l(\alpha, \beta, \lambda \mid \underline{r}, \underline{s})}{\partial \beta}=0$ and $\frac{\partial l(\alpha, \beta, \lambda \mid \underline{r}, \underline{s})}{\partial \lambda}=0$ simultaneously with respect to $\alpha, \beta$ and $\lambda$, where

$$
\begin{aligned}
\frac{\partial l(\alpha, \beta, \lambda \mid \underline{r}, \underline{s})}{\partial \alpha}= & \frac{n}{\alpha}+\ln \left[1-e^{-\lambda / r_{n}}\right] \\
\frac{\partial l(\alpha, \beta, \lambda \mid \underline{r}, \underline{s})}{\partial \beta}= & \frac{m}{\beta}+\ln \left[1-e^{-\lambda / s_{m}}\right] \\
\frac{\partial l(\alpha, \beta, \lambda \mid \underline{r}, \underline{s})}{\partial \lambda}= & \frac{n+m}{\lambda}+\frac{\alpha e^{-\lambda / r_{n}}}{r_{n}\left(1-e^{-\lambda / r_{n}}\right)}+\frac{\beta e^{-\lambda / s_{m}}}{s_{m}\left(1-e^{-\lambda / s_{m}}\right)}-\sum_{i=1}^{n} \frac{1}{r_{i}}-\sum_{i=1}^{n} \frac{e^{-\lambda / r_{i}}}{r_{i}\left(1-e^{-\lambda / r_{i}}\right)} \\
& -\sum_{i=1}^{m} \frac{1}{s_{i}}-\sum_{i=1}^{m} \frac{e^{-\lambda / s_{i}}}{s_{i}\left(1-e^{-\lambda / s_{i}}\right)}
\end{aligned}
$$


from equation (2.4) and (2.5) we get

$$
\begin{aligned}
& \hat{\alpha}_{M L}=h_{1}(\hat{\lambda})=\frac{n}{-\ln \left(1-e^{-\lambda / r_{n}}\right)} \\
& \hat{\beta}_{M L}=h_{2}(\hat{\lambda})=\frac{m}{-\ln \left(1-e^{-\lambda / s_{m}}\right)}
\end{aligned}
$$

and the MLE of $\lambda$ is the solution of the non-linear equation

$$
\frac{n+m}{\lambda}+\frac{h_{1}(\lambda) e^{-\lambda / r_{n}}}{r_{n}\left(1-e^{-\lambda / r_{n}}\right)}+\frac{h_{2}(\lambda) e^{-\lambda / s_{m}}}{s_{m}\left(1-e^{-\lambda / s_{m}}\right)}-\sum_{i=1}^{n} \frac{1}{r_{i}}\left(1+\frac{e^{-\lambda / r_{i}}}{\left(1-e^{-\lambda / r_{i}}\right)}\right)-\sum_{i=1}^{m} \frac{1}{s_{i}}\left(1+\frac{e^{-\lambda / s_{i}}}{\left(1-e^{-\lambda / s_{i}}\right)}\right)=0
$$

Hence, the MLE of $R$ based on upper records becomes

$$
\hat{R}_{M L}=\frac{\hat{\alpha}_{M L}}{\hat{\alpha}_{M L}+\hat{\beta}_{M L}}=\frac{1}{1+\left(\frac{m}{n}\right)\left(\frac{-\ln \left(1-e^{-\lambda / r_{n}}\right)}{-\ln \left(1-e^{-\lambda / s_{m}}\right)}\right)}
$$

From the above expression, it is very difficult to find the exact distribution of $\hat{R}_{M L}$. Therefore, we use another method to construct the CI of $R$ namely asymptotic distribution of MLE and parametric bootstrap method.

\section{CI for $R$}

In this Section, we discussed two different methods to obtain the confidence interval for $R$ namely the method of asymptotic normality and parametric bootstrap method.

\subsection{Asymptotic CI}

Here, we deduced the expression for asymptotic CI when $\lambda$ is unknown by using the multivariate delta method (see Wasserman, 2003, p.99). To compute the asymptotic distribution of $\hat{R}_{M L}$ we need to find an asymptotic variance of $\hat{R}_{M L}$. However, it is well known that the asymptotic variance is the inverse of the Fisher information matrix which is given as:

$$
I(\alpha, \beta, \lambda)=-E\left(\begin{array}{lll}
\frac{\partial^{2} L}{\partial \alpha^{2}} & \frac{\partial^{2} L}{\partial \alpha \partial \beta} & \frac{\partial^{2} L}{\partial \alpha \partial \lambda} \\
\frac{\partial^{2} L}{\partial \beta \partial \alpha} & \frac{\partial^{2} L}{\partial \beta^{2}} & \frac{\partial^{2} L}{\partial \beta \partial \lambda} \\
\frac{\partial^{2} L}{\partial \lambda \partial \alpha} & \frac{\partial^{2} L}{\partial \lambda \partial \beta} & \frac{\partial^{2} L}{\partial \lambda^{2}}
\end{array}\right)
$$

where,

$$
\begin{aligned}
& \frac{\partial^{2} L}{\partial \alpha^{2}}=-\frac{n}{\alpha^{2}}, \quad \frac{\partial^{2} L}{\partial \beta^{2}}=-\frac{m}{\beta^{2}}, \quad \frac{\partial^{2} L}{\partial \alpha \partial \beta}=\frac{\partial^{2} L}{\partial \beta \partial \alpha}=0, \\
& \frac{\partial^{2} L}{\partial \alpha \partial \lambda}=\frac{\partial^{2} L}{\partial \lambda \partial \alpha}=\frac{e^{-\lambda / r_{n}}}{r_{n}\left(1-e^{-\lambda / r_{n}}\right)}, \quad \frac{\partial^{2} L}{\partial \beta \partial \lambda}=\frac{\partial^{2} L}{\partial \lambda \partial \beta}=\frac{e^{-\lambda / s_{m}}}{s_{m}\left(1-e^{-\lambda / s_{m}}\right)}
\end{aligned}
$$


and

$$
\frac{\partial^{2} L}{\partial \lambda^{2}}=-\frac{(n+m)}{\lambda^{2}}-\frac{\alpha e^{-\lambda / r_{n}}}{r_{n}^{2}\left(1-e^{-\lambda / r_{n}}\right)^{2}}-\frac{\beta e^{-\lambda / s_{m}}}{s_{m}^{2}\left(1-e^{-\lambda / s_{m}}\right)^{2}}+\sum_{i=1}^{n} \frac{e^{-\lambda / r_{i}}}{r_{i}^{2}\left(1-e^{-\lambda / r_{i}}\right)^{2}}+\sum_{i=1}^{m} \frac{e^{-\lambda / s_{i}}}{s_{i}^{2}\left(1-e^{-\lambda / s_{i}}\right)^{2}}
$$

However, to find the expectation of the above defined terms are very complicated so, under some regularity conditions, we have used observed information matrix define as;

$$
\Phi(\alpha, \beta, \lambda)=-\left(\begin{array}{lll}
\frac{\partial^{2} L}{\partial \alpha^{2}} & \frac{\partial^{2} L}{\partial \alpha \partial \beta} & \frac{\partial^{2} L}{\partial \alpha \partial \lambda} \\
\frac{\partial^{2} L}{\partial \beta \partial \alpha} & \frac{\partial^{2} L}{\partial \beta^{2}} & \frac{\partial^{2} L}{\partial \beta \partial \lambda} \\
\frac{\partial^{2} L}{\partial \lambda \partial \alpha} & \frac{\partial^{2} L}{\partial \lambda \partial \beta} & \frac{\partial^{2} L}{\partial \lambda^{2}}
\end{array}\right)
$$

Using the multivariate delta method (Soliman et al. 2013) to find the approximate estimate of the asymptotic variance of $\hat{R}_{M L}, \hat{\Sigma}_{R}$ as follows:

Let $B^{\prime}=\left(\frac{\partial R}{\partial \alpha}, \frac{\partial R}{\partial \beta}, \frac{\partial R}{\partial \lambda}\right)$, where

$$
\frac{\partial R}{\partial \alpha}=\frac{\alpha}{(\alpha+\beta)^{2}}, \quad \frac{\partial R}{\partial \beta}=\frac{-\alpha}{(\alpha+\beta)^{2}} \text { and } \frac{\partial R}{\partial \lambda}=0
$$

Therefore $\hat{\Sigma}_{R} \cong\left[B^{\prime} \Phi^{-1} B\right]_{\hat{\alpha}_{M L}, \hat{\beta}_{M L}, \hat{\lambda}_{M L}}$ The asymptotic distribution of $\hat{R}_{M L}$ is $N\left(R, \hat{\Sigma}_{R}\right)$ and it can also be written as :

$$
\frac{\hat{R}_{M L}-R}{\sqrt{\hat{\Sigma}_{R}}} \stackrel{d}{\longrightarrow} N(0,1)
$$

where $N(a, b)$ denotes normal distribution with mean $a$ and variance $b$ and the symbol $\stackrel{d}{\longrightarrow}$ denotes the convergence in distribution. Based on this asymptotic distribution, a $100(1-\gamma) \%$ asymptotic CI for $R$ is given by

$$
\hat{R}_{M L}+Z_{\gamma / 2} \sqrt{\hat{\Sigma}_{R}}
$$

Where $Z_{\gamma / 2}$ denotes upper $\gamma / 2$ quantile value of $N(0,1)$.

\subsection{Parametric bootstrap CI}

Here, we have constructed a bootstrap CI for $R$ by using a parametric percentile bootstrap method (Efron. 1982). The following algorithm is used to generate the parametric bootstrap estimates of $R$.

Step-1. Simulate a random sample from Uniform $(0,1)$. Using this simulated value compute random sample for $X \sim \operatorname{GIED}(\lambda, \alpha)$ and $y \sim \operatorname{GIED}(\lambda, \beta)$ respectively. Compute the MLE of $\alpha, \beta$ and $\lambda$ say $\hat{\alpha}_{M L}, \hat{\beta}_{M L}$ and $\hat{\lambda}_{M L}$ given in section- 2 .

Step-2. Generate an independent parametric bootstrap sample using $\hat{\alpha}_{M L}, \hat{\beta}_{M L}$ and $\hat{\lambda}_{M L}$ instead of $\alpha, \beta$ and $\lambda$. then using these values, calculate $\hat{R}_{M L}$.

Step-3. Calculate the maximum likelihood estimate of $\hat{\alpha}_{M L}, \hat{\beta}_{M L}, \hat{\lambda}_{M L}$ and $\hat{R}_{M L}$ obtained in step-2 say $\hat{\alpha}_{M L}^{\prime}, \hat{\beta}_{M L}^{\prime}, \hat{\lambda}_{M L}^{\prime}$ and $\hat{R}_{M L}^{\prime}$. 
Step-4. Repeat the step-2 and step-3 $N$ times to obtained the parametric bootstrap estimates $\hat{R}_{M L 1}^{\prime}, \hat{R}_{M L 2}^{\prime}, \ldots, \hat{R}_{M L N}^{\prime}$ of $R$.

Step-5. Let $H(x)=P\left(\hat{R}_{M L} \leq x\right)$ be the cumulative distribution function of $\hat{R}_{M L}$. Define $\hat{R}_{\text {Boot }}(x)=H^{-1}(x)$ for a given $x$. The approximate $100(1-\gamma) \%$ CI of $R$ is given by $\left(\hat{R}_{\text {Boot }}(\gamma / 2), \hat{R}_{\text {Boot }}(1-\gamma / 2)\right)$.

\section{UMVUE of $R$}

In this Section, we have derived the expression for UMVUE of $R$ based on upper record values when the observations follow GIED with a common and known parameter $\lambda$. The technique used for obtaining the UMVUE of $R$ is similar to Khan and Arshad (2016).

Let $X_{1}, X_{2}, \ldots$ be a sequence of iid random variables from $\operatorname{GIED}(\lambda, \alpha)$ indicating the stress in a reliability system and $r=r_{1}, r_{2}, \ldots, r_{n}$ be the induced upper records from this sequence of random variables, then the joint $p d f$ of $n$ upper records is given by

$$
f(\lambda, \alpha \mid \underline{r})=(\alpha \lambda)^{n}\left(1-e^{-\lambda / r_{n}}\right)^{\alpha} \prod_{i=1}^{n} \frac{e^{-\lambda / r_{i}}}{r_{i}^{2}\left(1-e^{-\lambda / r_{i}}\right)}, \quad r_{1}<r_{2}<\ldots<r_{n}
$$

Similarly, let $Y_{1}, Y_{2}, \ldots$ be another sequence of iid random variables from $\operatorname{GIED}(\lambda, \beta)$ indicating the strength in reliability system and $\underline{s}=s_{1}, s_{2}, \ldots, s_{m}$ be the induced upper records from this sequence of random variables, then the joint $p d f$ of $m$ upper records is given by

$$
f(\lambda, \beta \mid \underline{s})=(\beta \lambda)^{m}\left(1-e^{-\lambda / s_{m}}\right)^{\beta} \prod_{i=1}^{m} \frac{e^{-\lambda / s_{i}}}{s_{i}^{2}\left(1-e^{-\lambda / s_{i}}\right)}, \quad s_{1}<s_{2}<\ldots<s_{m}
$$

To obtain the UMVUE of $R$, we need an unbiased and complete sufficient statistics of $\alpha$ and $\beta$. Let $U_{1}=-\ln \left(1-e^{-\lambda / r_{1}}\right)$ and $V_{1}=-\ln \left(1-e^{-\lambda / s_{1}}\right)$, then the indicator function defined as

$$
I\left(U_{1}<V_{1}\right)= \begin{cases}1, & \text { if } U_{1}<V_{1} \\ 0, & \text { otherwise }\end{cases}
$$

is an unbiased estimator for $R$. From equation (4.1) and (4.2), it can be seen that $U_{n}=-\ln \left(1-e^{-\lambda / r_{n}}\right)$ and $V_{m}=-\ln \left(1-e^{-\lambda / s_{m}}\right)$ be the complete sufficient statistics for $\alpha$ and $\beta$ and have gamma distribution with parameters $(n, \alpha)$ and $(m, \beta)$ respectively. By the application of Lehman - Scheff $\ddot{e}$ theorem (Lehmann and Casella (1998)), the UMVUE of $R$ is given by

$$
\begin{aligned}
\hat{R}_{U M} & =E\left[I\left(U_{1}<V_{1}\right) \mid U_{n}=u_{n}, V_{m}=v_{m}\right] \\
& =\iint_{C} f_{U_{1} \mid U_{n}=u_{n}}\left(u_{1} \mid u_{n}\right) f_{V_{1} \mid V_{m}=v_{m}}\left(v_{1} \mid v_{m}\right) d u_{1} d v_{1}
\end{aligned}
$$

.where $C=\left(u_{1}, v_{1}\right) ; 0<u_{1}<u_{n}, 0<v_{1}<v_{m}, u_{1}<v_{1}$.

Before solving the above integral, it is required to find the conditional distribution of $U_{1} \mid U_{n}$ and $V_{1} \mid V_{m}$. By some algebraic simplification, we get. 


$$
f_{U_{1} \mid U_{n}=u_{n}}\left(u_{1} \mid u_{n}\right)=(n-1) \frac{\left(u_{n}-u_{1}\right)^{n-2}}{u_{n}^{n-1}}, \quad 0<u_{1}<u_{n}
$$

and

$$
f_{V_{1} \mid V_{m}=v_{m}}\left(v_{1} \mid v_{m}\right)=(m-1) \frac{\left(v_{m}-v_{1}\right)^{m-2}}{v_{m}^{m-1}}, \quad 0<v_{1}<v_{m}
$$

Therefore, the UMVUE of $R=P(X<Y)$ is given by

$$
\begin{aligned}
\hat{R}_{U M} & =\int_{0}^{\min \left(u_{n}, v_{m}\right)} \int_{u_{1}}^{v_{m}}(n-1) \frac{\left(u_{n}-u_{1}\right)^{n-2}}{u_{n}^{n-1}}(m-1) \frac{\left(v_{m}-v_{1}\right)^{m-2}}{v_{m}^{m-1}} d v_{1} d u_{1} \\
& =\frac{(n-1)}{u_{n}} \int_{0}^{\min \left(u_{n}, v_{m}\right)}\left(1-\frac{u_{1}}{u_{n}}\right)^{n-2}\left(1-\frac{u_{1}}{v_{m}}\right)^{m-1} d u_{1}
\end{aligned}
$$

Expanding the term $\left(1-\frac{u_{1}}{v_{m}}\right)^{m-1}$ binomially, we get

$$
\left.\hat{R}_{U M}=\frac{(n-1)}{u_{n}} \sum_{p=0}^{m-1}(-1)^{p}\left(\begin{array}{c}
m-1 \\
p
\end{array}\right)^{\min \left(u_{n}, v_{m}\right)} \int_{0}^{n-\frac{u_{1}}{u_{n}}}\right)^{n-2}\left(\frac{u_{1}}{v_{m}}\right)^{p} d u_{1}
$$

Taking transformation $z=\frac{u_{1}}{u_{n}}$ and simplifying simultaneously, we get

$$
\hat{R}_{U M}=\frac{(n-1)}{u_{n}} \sum_{p=0}^{m-1}(-1)^{p}\left(\begin{array}{c}
m-1 \\
p
\end{array}\right)\left(\frac{u_{n}}{v_{m}}\right)^{p} \int_{0}^{\min \left(1, v_{m} / u_{n}\right)}(1-z)^{n-2} z^{p} d z
$$

Here, it can be seen that the integral value depends on the value of $u_{n}$ and $v_{m}$. Hence, there arise two cases, $u_{n} \leq v_{m}$ and $u_{n}>v_{m}$.

Case-I: When $u_{n} \leq v_{m}$. In this case,

$$
\hat{R}_{U M}=(n-1) \sum_{p=0}^{m-1}(-1)^{p}\left(\begin{array}{c}
m-1 \\
p
\end{array}\right)\left(\frac{u_{n}}{v_{m}}\right)^{p} B(p+1, n-1)
$$

where, $B(a, b)=\int_{0}^{1} x^{a-1}(1-x)^{b-1} d x$ is the complete beta function.

Case-II: when $u_{n}>v_{m}$, we have

$$
\hat{R}_{U M}=(n-1) \sum_{p=0}^{m-1}(-1)^{p}\left(\begin{array}{c}
m-1 \\
p
\end{array}\right)\left(\frac{u_{n}}{v_{m}}\right)^{p} B_{\frac{v_{m}}{u_{n}}}(p+1, n-1)
$$

where, $B_{v}(a, b)=\int_{0}^{v} x^{a-1}(1-x)^{b-1} d x$ denotes the incomplete beta function with upper limit $v$. Using the relation of incomplete beta and Gauss hyper-geometric function $B_{v}(a, b)=\frac{v^{a}}{a}{ }_{2} F_{1}(a, 1-b ; a+1 ; v)$, equation (4.6) can also be written as 


$$
\hat{R}_{U M}=\frac{(n-1)}{m}\left(\frac{v_{m}}{u_{n}}\right) \sum_{p=0}^{m-1}(-1)^{p}\left(\begin{array}{c}
m-1 \\
p
\end{array}\right){ }_{2} F_{1}\left(p+1,2-n ; p+2 ; \frac{v_{m}}{u_{n}}\right)
$$

where ${ }_{2} F_{1}(a, 1-b ; a+1 ; v)$ is the Gauss hyper-geometric function.

\section{Bayesian Estimation}

This section presents a study of Bayesian estimation of $R$. We know that in Bayesian inference, we need some prior distributions for unknown parameters of parent distribution. we have considered gamma distributions as a prior distribution for $\alpha, \beta$ and $\lambda$ with $p d f$ given as

$$
\begin{array}{ll}
\psi(\alpha)=\frac{\delta_{1}^{\eta_{1}}}{\Gamma \eta_{1}} \alpha^{\eta_{1}-1} e^{-\delta_{1} \alpha}, & 0<\alpha<\infty, \delta_{1}, \eta_{1}>0 \\
\psi(\beta)=\frac{\delta_{2}^{\eta_{21}}}{\Gamma \eta_{2}} \beta^{\eta_{2}-1} e^{-\delta_{2} \beta}, & 0<\beta<\infty, \delta_{2}, \eta_{2}>0
\end{array}
$$

and

$$
\psi(\lambda)=\frac{\delta_{3}^{\eta_{3}}}{\Gamma \eta_{3}} \lambda^{\eta_{3}-1} e^{-\delta_{3} \lambda}, \quad 0<\lambda<\infty, \delta_{3}, \eta_{3}>0
$$

where $\left(\eta_{1}, \delta_{1}\right),\left(\eta_{2}, \delta_{2}\right)$ and $\left(\eta_{3}, \delta_{3}\right)$ are hyper-parameters chosen to reflect prior knowledge about $\alpha, \beta$ and $\lambda$.

From (2.2), the likelihood function can be re-written as

$$
L(\alpha, \beta, \lambda \mid \underline{r}, \underline{s})=\alpha^{n} \beta^{m} \lambda^{n+m} e^{-\alpha\left[-\ln \left(1-e^{-\lambda / r_{n}}\right)\right]} e^{-\beta\left[-\ln \left(1-e^{-\lambda / s_{m}}\right)\right]} \varphi(\lambda \mid \underline{r}) \varphi(\lambda \mid \underline{s})
$$

where $\varphi(\lambda \mid \underline{r})=\prod_{i=1}^{n} \frac{e^{-\lambda / r_{i}}}{r_{i}^{2}\left(1-e^{-\lambda / r_{i}}\right)}$ and $\varphi(\lambda \mid \underline{s})=\prod_{i=1}^{m} \frac{e^{-\lambda / s_{i}}}{s_{i}^{2}\left(1-e^{-\lambda / s_{i}}\right)}$.

In order to find the posterior distribution of $R$, we need to obtain joint posterior distribution of $\alpha, \beta$ and $\lambda$. Using Bayes' theorem (Wasserman, 2003).

From (5.1), (5.2), (5.3) and (5.4) the joint posterior distribution of $\alpha, \beta$ and $\lambda$ is given by

$$
\pi(\alpha, \beta, \lambda \mid \underline{r}, \underline{s})=\frac{\psi(\alpha) \psi(\beta) \psi(\lambda) f(\alpha, \beta, \lambda \mid \underline{r}, \underline{s})}{\int_{0}^{\infty} \int_{0}^{\infty} \int_{0}^{\infty} \psi(\alpha) \psi(\beta) \psi(\lambda) f(\alpha, \beta, \lambda \mid \underline{r}, \underline{s}) d \alpha d \beta d \lambda}
$$

after solving the above expression, we get

$$
\begin{aligned}
\pi(\alpha, \beta, \lambda \mid \underline{r}, \underline{s}) \propto & \alpha^{n+\eta_{1}-1} \beta^{m+\eta_{2}-1} \lambda^{n+m+\eta_{3}-1} e^{-\alpha\left[\delta_{1}-\ln \left(1-e^{-\lambda / r_{n}}\right)\right]} e^{-\beta\left[\delta_{2}-\ln \left(1-e^{-\lambda / s_{m}}\right)\right]} e^{-\lambda \delta_{3}} \\
& \varphi(\lambda \mid \underline{r}) \varphi(\lambda \mid \underline{s})
\end{aligned}
$$

From the above equation, the joint posterior is very complicated and hence it is not possible to obtain a closed form or explicit expression for Bayes estimator of $R$. Therefore, to simulate the samples from the posterior distribution, we have considered the MCMC approach to find a sample based inferences. Solimon et al. (2013) considered the MCMC approach for stress-strength reliability model for the complete sample using modified weibull distribution.

\section{Simulation Study}


In this section, a Monte Carlo simulation study is conducted to justify the performance of all estimators presented in the preceding sections for different sample sizes and different shape parameter values. Upper records sizes $(n, m)=(5,5),(5,8),(8,5)$ and $(8,8)$ are considered and suppose $\alpha=\lambda=1$ and changing the value of $\beta$ in such a way that we can obtained $R=0.1,0.2, \ldots, 0.9$. In Bayesian estimation, we have taken an informative prior function by choosing the values of hyper-parameters as $\left(\eta_{1}, \eta_{2}, \delta_{1}, \delta_{2}\right)=(1,1,1,1)$. 10,000 replications are used for classical approache while we have taken 1000 repetition for Bayeian approach.The average biases and mean squared errors (MSE) for MLE and Bayes estimator and MSE for UMVUE are noted which are shown in Table-1. Moreover, to observe the behavior of different CIs in terms of different sample sizes and different parameter values, we obtained $95 \%$ coverage probability $(\mathrm{CP})$ and expected width (EW) of various CIs and Bayesian credible interval which are given in Table- 2 .

Table-1: MSE of UMVUE and Bias and MSE of MLE, Bayes estimator of $R$

\begin{tabular}{|c|c|c|c|c|c|c|c|c|}
\hline \multirow[b]{2}{*}{$\alpha$} & \multirow[b]{2}{*}{$\beta$} & \multirow[b]{2}{*}{$R$} & \multirow[b]{2}{*}{$(\mathbf{n}, \mathbf{m})$} & \multicolumn{2}{|c|}{ MLE } & \multirow{2}{*}{$\begin{array}{l}\text { UMVUE } \\
\text { MSE }\end{array}$} & \multicolumn{2}{|c|}{ Bayes Estimator } \\
\hline & & & & Bias & MSE & & Bias & MSE \\
\hline 1 & \multirow[t]{4}{*}{9} & \multirow[t]{4}{*}{0.1} & $(5,5)$ & 0.0008 & 0.0000006 & 0.0000004 & -0.1170856 & 0.0137090 \\
\hline & & & $(8,5)$ & 0.0018 & 0.0000034 & 0.0000009 & -0.0876903 & 0.0076896 \\
\hline & & & $(5,8)$ & 0.0023 & 0.0000052 & 0.0000703 & -0.1338278 & 0.0179099 \\
\hline & & & $(8,8)$ & -0.0004 & 0.0000021 & 0.0000014 & -0.0923987 & 0.0085375 \\
\hline & \multirow[t]{4}{*}{4} & \multirow[t]{4}{*}{0.2} & $(5,5)$ & 0.0034 & 0.0000112 & 0.0000079 & -0.0768704 & 0.0059091 \\
\hline & & & $(8,5)$ & 0.0032 & 0.0000102 & 0.0000013 & -0.0578596 & 0.0033477 \\
\hline & & & $(5,8)$ & 0.0012 & 0.0000013 & 0.0000280 & -0.1001602 & 0.0100321 \\
\hline & & & $(8,8)$ & 0.0023 & 0.0000052 & 0.0000024 & -0.0591719 & 0.0035013 \\
\hline & \multirow[t]{4}{*}{2.33} & \multirow[t]{4}{*}{0.3} & $(5,5)$ & 0.0018 & 0.0000032 & 0.0000027 & -0.0412098 & 0.0016983 \\
\hline & & & $(8,5)$ & 0.0030 & 0.0000088 & 0.0000047 & -0.0528431 & 0.0027924 \\
\hline & & & $(5,8)$ & 0.0038 & 0.0000145 & 0.0000167 & -0.0532959 & 0.0028405 \\
\hline & & & $(8,8)$ & -0.0010 & 0.0000011 & 0.0000006 & -0.0459495 & 0.0021114 \\
\hline & \multirow[t]{4}{*}{1.5} & \multirow[t]{4}{*}{0.4} & $(5,5)$ & -0.0055 & 0.0000307 & 0.0000120 & -0.0043593 & 0.0000190 \\
\hline & & & $(8,5)$ & -0.0018 & 0.0000032 & 0.0000131 & -0.0166388 & 0.0002768 \\
\hline & & & $(5,8)$ & 0.0040 & 0.0000157 & 0.0000084 & -0.0378956 & 0.0014361 \\
\hline & & & $(8,8)$ & 0.0020 & 0.0000039 & 0.0000012 & -0.0687202 & 0.0047225 \\
\hline & \multirow[t]{4}{*}{1} & \multirow[t]{4}{*}{0.5} & $(5,5)$ & -0.0074 & 0.0000542 & 0.0000317 & -0.0086856 & 0.0000754 \\
\hline & & & $(8,5)$ & 0.0053 & 0.0000276 & 0.0000325 & -0.0031019 & 0.0000096 \\
\hline & & & $(5,8)$ & 0.0047 & 0.0000225 & 0.0000140 & 0.0152529 & 0.0002327 \\
\hline & & & $(8,8)$ & -0.0021 & 0.0000046 & 0.0000013 & -0.0037939 & 0.0000144 \\
\hline & \multirow[t]{4}{*}{0.67} & \multirow[t]{4}{*}{0.6} & $(5,5)$ & -0.0037 & 0.0000143 & 0.0000128 & 0.0164605 & 0.0002709 \\
\hline & & & $(8,5)$ & -0.0053 & 0.0000387 & 0.0000156 & 0.0152787 & 0.0002334 \\
\hline & & & $(5,8)$ & -0.0060 & 0.0000358 & 0.0000050 & 0.5844009 & 0.0002074 \\
\hline & & & $(8,8)$ & 0.0028 & 0.0000138 & 0.0000044 & 0.0132183 & 0.0001747 \\
\hline & \multirow[t]{4}{*}{0.43} & \multirow[t]{4}{*}{0.7} & $(5,5)$ & 0.0018 & 0.0000034 & 0.0000022 & 0.0442016 & 0.0019538 \\
\hline & & & $(8,5)$ & -0.0054 & 0.0000297 & 0.0000049 & 0.0389809 & 0.0015195 \\
\hline & & & $(5,8)$ & -0.0092 & 0.0000849 & 0.0000026 & 0.0428114 & 0.0018328 \\
\hline & & & $(8,8)$ & 0.0023 & 0.0000054 & 0.0000011 & 0.0326244 & 0.0010644 \\
\hline & \multirow[t]{4}{*}{0.25} & \multirow[t]{4}{*}{0.8} & $(5,5)$ & -0.0013 & 0.0000046 & 0.0000058 & 0.0561747 & 0.0031556 \\
\hline & & & $(8,5)$ & 0.0020 & 0.0000040 & 0.0000015 & 0.0441383 & 0.0019481 \\
\hline & & & $(5,8)$ & -0.0017 & 0.0000029 & 0.0000025 & 0.0506424 & 0.0025647 \\
\hline & & & $(8,8)$ & 0.0041 & 0.0000098 & 0.0000036 & 0.0371154 & 0.0013776 \\
\hline & \multirow[t]{4}{*}{0.11} & \multirow[t]{4}{*}{0.9} & $(5,5)$ & -0.0007 & 0.0000006 & 0.0000004 & 0.0465354 & 0.0021655 \\
\hline & & & $(8,5)$ & 0.0009 & 0.0000008 & 0.0000005 & 0.0349021 & 0.0012182 \\
\hline & & & $(5,8)$ & -0.0030 & 0.0000087 & 0.0000004 & 0.0408159 & 0.0016659 \\
\hline & & & $(8,8)$ & 0.0002 & 0.0000009 & 0.0000006 & 0.0269263 & 0.0007250 \\
\hline
\end{tabular}


Table-2: $\mathrm{EW}$ and $\mathrm{CP}$ of confidence interval (CI) for various interval estimates of $R$ with

\begin{tabular}{|c|c|c|c|c|c|c|c|c|c|}
\hline \multirow[b]{2}{*}{$R$} & \multirow[b]{2}{*}{$(\mathbf{n}, \mathbf{m})$} & \multicolumn{2}{|c|}{ MLE } & \multicolumn{2}{|c|}{ Boot } & \multicolumn{2}{|c|}{ Asymptotic } & \multicolumn{2}{|c|}{ Bayes Estimator } \\
\hline & & EWCI & $\mathbf{C P}$ & EWCI & $\mathbf{C P}$ & EWCI & $\mathbf{C P}$ & EWCI & $\mathbf{C P}$ \\
\hline \multirow[t]{4}{*}{0.1} & $(5,5)$ & 0.3260 & 0.974 & 0.3202 & 0.969 & 0.2102 & 0.938 & 0.3779 & 0.884 \\
\hline & $(8,5)$ & 0.3362 & 0.887 & 0.3424 & 0.906 & 0.2167 & 0.940 & 0.3633 & 0.771 \\
\hline & $(5,8)$ & 0.1353 & 0.873 & 0.2921 & 0.885 & 0.1706 & 0.930 & 0.3011 & 0.761 \\
\hline & $(8,8)$ & 0.2433 & 0.980 & 0.2271 & 0.970 & 0.1748 & 0.941 & 0.2824 & 0.932 \\
\hline \multirow[t]{4}{*}{0.2} & $(5,5)$ & 0.4219 & 0.971 & 0.4545 & 0.971 & 0.3867 & 0.946 & 0.4370 & 0.759 \\
\hline & $(8,5)$ & 0.3811 & 0.870 & 0.5089 & 0.902 & 0.3869 & 0.954 & 118 & 0.891 \\
\hline & $(5,8)$ & 0.3100 & 0.892 & 0.3291 & 0.883 & 0.3082 & 0.945 & 0.3684 & 0.667 \\
\hline & $(8,8)$ & 0.3655 & 0.975 & 0.3478 & 0.978 & 0.3069 & 0.953 & 0.3393 & 0.877 \\
\hline \multirow[t]{4}{*}{0.3} & $(5,5)$ & 0.5405 & 0.976 & 0.5865 & 0.965 & 0.5122 & 0.962 & 0.4825 & 0.900 \\
\hline & $(8,5)$ & 0.5625 & 0.874 & 0.5728 & 0.875 & 0.5111 & 0.969 & 0.4440 & 0.891 \\
\hline & $(5,8)$ & 0.4204 & 0.898 & 0.3095 & 0.882 & 0.4034 & 0.955 & 0.4337 & 0.834 \\
\hline & $(8,8)$ & 0.2214 & 0.981 & 0.3716 & 0.979 & 0.4071 & 0.961 & 0.3931 & 0.919 \\
\hline \multirow[t]{4}{*}{0.4} & $(5,5)$ & 0.6234 & 0.966 & 0.5390 & 0.969 & 0.5836 & 0.966 & 0.5108 & 0.892 \\
\hline & $(8,5)$ & 0.5303 & 0.878 & 0.5696 & 0.891 & 0.5877 & 0.978 & 0.4682 & 0.92 \\
\hline & $(5,8)$ & 0.5292 & 0.885 & 0.5718 & 0.864 & 0.4678 & 0.948 & 0.4607 & 0.876 \\
\hline & $(8,8)$ & 0.4896 & 0.967 & 0.4717 & 0.973 & 0.4661 & 0.967 & 0.4266 & 0.918 \\
\hline \multirow[t]{4}{*}{0.5} & $(5,5)$ & 0.6195 & 0.978 & 0.6306 & 0.971 & 0.6119 & 0.969 & 0.5088 & 0.963 \\
\hline & $(8,5)$ & 0.5683 & 0.873 & 0.4969 & 0.889 & 0.6118 & 0.987 & 0.4746 & 0.821 \\
\hline & $(5,8)$ & 0.5041 & 0.887 & 0.5423 & 0.890 & 0.4837 & 0.950 & 0.4700 & 0.804 \\
\hline & $(8,8)$ & 0.4921 & 0.983 & 0.4829 & 0.969 & 0.4837 & 0.971 & 0.4299 & 0.968 \\
\hline \multirow[t]{4}{*}{0.6} & $(5,5)$ & 0.6072 & 0.971 & 0.6111 & 0.972 & 0.5856 & 0.968 & 0.4960 & 0.925 \\
\hline & $(8,5)$ & 0.5673 & 0.890 & 0.5556 & 0.867 & 0.5881 & 0.981 & 644 & 0.818 \\
\hline & $(5,8)$ & 0.5648 & 0.876 & 0.5481 & 0.884 & 0.4634 & 0.939 & 0.4569 & 0.907 \\
\hline & $(8,8)$ & 0.3870 & 0.972 & 0.4964 & 0.974 & 0.4651 & 0.970 & 0.4182 & 0.914 \\
\hline \multirow[t]{4}{*}{0.7} & $(5,5)$ & 0.5916 & 0.982 & 0.5862 & 0.976 & 0.5158 & 0.954 & 0.4617 & 0.798 \\
\hline & $(8,5)$ & 0.4291 & 0.884 & 0.4746 & 0.890 & 0.5086 & 0.972 & 0.4296 & 0.892 \\
\hline & $(5,8)$ & 0.5726 & 0.914 & 0.5451 & 0.883 & 0.4021 & 0.929 & 0.4236 & 0.789 \\
\hline & $(8,8)$ & 0.3373 & 0.974 & 0.4945 & 0.978 & 0.4081 & 0.955 & 0.3837 & 0.876 \\
\hline \multirow[t]{4}{*}{0.8} & $(5,5)$ & 0.6050 & 0.970 & 0.5542 & 0.976 & 0.3898 & 0.941 & 0.3977 & 0.892 \\
\hline & $(8,5)$ & 0.3398 & 0.878 & 0.2419 & 0.901 & 0.3946 & 0.963 & 0.3634 & 0.826 \\
\hline & $(5,8)$ & 0.5493 & 0.885 & 0.4742 & 0.869 & 0.3076 & 0.922 & 0.3591 & 0.743 \\
\hline & $(8,8)$ & 0.4352 & 0.973 & 0.3561 & 0.977 & 0.3177 & 0.948 & 0.3186 & 0.789 \\
\hline \multirow[t]{4}{*}{0.9} & $(5,5)$ & 0.5378 & 0.963 & 0.4538 & 0.969 & 0.2188 & 0.931 & 0.2720 & 0.924 \\
\hline & $(8,5)$ & 0.2545 & 0.881 & 0.2605 & 0.898 & 0.2221 & 0.902 & 0.2403 & 0.843 \\
\hline & $(5,8)$ & 0.3253 & 0.879 & 0.3622 & 0.887 & 0.1696 & 0.907 & 0.2390 & 0.761 \\
\hline & $(8,8)$ & 0.1686 & 0.980 & 0.1287 & 0.971 & 0.1744 & 0.939 & 0.2041 & 0.918 \\
\hline
\end{tabular}

From the results given in tables 1 and 2, we conclude that;

- From Table-1, it is observed that MSE (MLE) > MSE (UMVUE) i.e. UMVUE perform better than MLE in the sense of MSE. In case of Bayes estimator, biases are negative (positive) when $R<0.5(R>0.5)$. Also, it can be seen that when the sample size increases, MSE of all the estimators decreases which is obvious. 
- From Table-2, we observed that the EWCI for asymptotic distribution is less than the others and the CPs for the asymptotic distribution are less than the nominal level 0.95 while in other cases (MLE and bootstrap), CPs are greater than the nominal level 0.95. When $R=0.5$, The width of the CIs is maximum and when moving to the extremes it gets reduces. When sample size increases, the length of the intervals decreases.

\section{Real data application}

Consider a real data set as an application of the estimation method described in this paper. This data is a champion's league data in which the first goal scoring in minutes during the final stages matches in two consecutive years (2011-2012 and 2012-2013) are considered separately for the return matches and the first matches of the European Champion League. This data is available online at http://www.it.soccerway.com and used by Condino et al. (2018). The data-set is given below:

We have considered only upper record values by taking the larger than the preceding largest. There are 8 upper records $(0.033,0.111,0.344,0.622,0.633,0.822,0.833,0.956)$ in data $X$ and 4 upper records $(0.267,0.611,0.711,0.922)$ in data Y. Using the Kolmogorov-Smirnov (K-S) test, we conclude that

Table-3: K-S statistics with distribution parameters Based on real data set.

\begin{tabular}{lcc}
\hline & K-S statistics & p-value \\
\hline $\mathrm{X} \sim \operatorname{GIED}(1,0.6)$ & 0.25 & 0.9801 \\
$\mathrm{Y} \sim \operatorname{GIED}(1,0.3)$ & 0.26 & 0.9739
\end{tabular}

where, $\operatorname{GIED}(\overline{a, b)}$ denotes the generalized inverted exponential distribution with parameter $a$ and $b$ and the calculated value of $R$ is 0.6667 . The largest upper record value from data $\mathrm{X}$ and data set $\mathrm{Y}$ are 0.956 and 0.922 respectively. From the upper record data, we have $(\mathrm{n}, \mathrm{m})=(8,4), u_{n}=0.956$ and $v_{m}=0.922$. From (2.7) and (4.6), the MLE and UMVUE of $R$ are 0.6559 and 0.6914 respectively and the $95 \%$ corresponding confidence interval is $(0.2568,0.8650)$. Further, from (5.6), the Bayes estimators for different choices of hyperparameters $\left(\eta_{1}, \eta_{2}, \delta_{1}, \delta_{2}\right)$ of prior distributions and corresponding $95 \%$ credible intervals are shown in table-4.

Table-4: Bayes estimators and respective $95 \%$ credible intervals based on real data.

\begin{tabular}{ccc}
\hline$\left(\eta_{1}, \eta_{2}, \delta_{1}, \delta_{2}\right)$ & $\hat{R}_{\text {Bayes }}$ & Credible Interval \\
\hline$(1,1,1,1)$ & 0.6383 & $(0.3808,0.8588)$ \\
$(1 / 2,1 / 2,1,1)$ & 0.6494 & $(0.3826,0.8731)$ \\
$(1,1,1 / 2,1 / 2)$ & 0.6361 & $(0.3785,0.8576)$ \\
$(1 / 2,1 / 2,1 / 2,1 / 2)$ & 0.6473 & $(0.3803,0.8720)$ \\
$(0,0,0,0)$ & 0.6598 & $(0.3863,0.8862)$ \\
\hline
\end{tabular}

\section{Conclusions}

In this paper, we have obtained the MLE, UMVUE and Bayesian estimator of $R=P(X<Y)$ from GIED with a common scale and different shape parameters based on upper record values. We have obtained the Bayes estimator of $R$ by using squared error loss function. Asymptotic CI, Bootstrap CI using parametric percentile bootstrap method and the Bayesian credible interval are discussed. Further, a simulation study is being 
carried out to compare the performances of MLE and UMVUE and observed that UMVUE perform better than MLE in the sense of MSE. Moreovr, it is noticed that asymptotic CI provided the smallest average width of CI for different sample sizes as compare to MLE and bootstrap CIs.

\section{Acknowledgment}

The authors would like to thank the chief editor, editor and referees for helpful comments and valuable suggestions which greatly help to improve the quality of this manuscript. The second author acknowledges with thanks to the University Grand Commission, India for financial support for this research work under a grant MANF-2014-15-MUS-UTT41028.

\section{References}

1. Abouammoh, A. M., Alshingiti, A. M. (2009). Reliability of generalized inverted exponential distribution. J. Stat. Comput. Simul., 79(11), 1301-1315.

2. Ahmadi, J. and Arghami, N. R. (2003a). Comparing the Fisher information in record values and iid observations. Statistics, 37(5), 435-441.

3. Ahmadi, J. and Arghami, N. R. (2003b). Non parametric confidence and tolerance intervals from record values data. Stat. Papers, 44, 455-468.

4. Ahsanullah, M. (1995). Introduction to record statistics, NOVA Science Publishers Inc., Hunting ton, New York.

5. Amin, E. A. (2017). Estimation of Stress-Strength Reliability for Kumaraswamy Exponential Distribution based on Upper Record Values. International Journal of Contemporary Mathematical Sciences \}, 12(2), 59-71.

6. Arnold, B. C., Balakrishnan, N. and Nagaraja, H. N. (1998). Records. Wiley, New York.

7. Awad, A. M., Azzam, M. M. and Hamadan, M. A. (1981). Some inference results in $\mathrm{P}(\mathrm{Y}<\mathrm{X})$ in the bivariate exponential model. Comm. Statis. Theory Methods, 10(24), 2515-2524.

8. Baklizi, A. (2008a). Estimation of $\mathrm{P}(\mathrm{X}<\mathrm{Y})$ using record values in one and two parameter exponential distributions. Comm. Statis. Theory Methods, 37(5), 692698.

9. Baklizi, A. (2008b). Likelihood and Bayesian estimation of $\mathrm{P}(\mathrm{X}<\mathrm{Y})$ using lower record values from the generalized exponential distribution. Comput. Statist. Data Anal., 52(7), 3468-3473.

10. Baklizi, A. (2014a). Bayesian inference for $\operatorname{Pr}(\mathrm{Y}<\mathrm{X})$ in the exponential distribution based on records. Appl. Math. Model, 38(5-6), 1698-1709.

11. Baklizi, A. (2014b). Interval estimation of the stress-strength reliability in the two-parameter exponential distribution based on records. J. Stat. Comput. Simul., 84(12), 2670-2679.

12. Basirat, M., Baratpour, S. and Ahmadi, J. (2016). On estimation of stress-strength parameter using record values from proportional hazard rate models. Comm. Statis. Theory Methods, 45(19), 5787-5801.

13. Condino, F., Domma, F. and Latorre, G. (2018). Likelihood and Bayesian estimation of $\mathrm{P}(\mathrm{Y}<\mathrm{X})$ using lower record values from a proportional reversed hazard family. Stat. Papers, 59(2), 467-485. 
14. Chandler, K. N. (1952). The distribution and frequency of record values. J. R. Statist. Soc., B, 14(2), 220-228.

15. Dey, S. (2007). Inverted exponential distribution as a life distribution model from a Bayesian viewpoint. Data Sci. J., 6(4), 107-113.

16. Dey, S. and Dey, T. (2014a). Generalized Inverted Exponential Distribution: Different Methods of Estimation, Amer. J. Math. Management Sci., 33(3), 194215.

17. Dey, S. and Dey, T. (2014b). On progressively censored generalized inverted exponential distribution, J. Appl. Stat., 41(12), 2557-2576,

18. Dey, S., Pradhan, B. (2014). Generalized inverted exponential distribution under hybrid censoring. Stat. Methodol. 18, 101-114.

19. Dey, S. and Dey, T. and Luckettc, D. J. (2016). Statistical inference for the generalized inverted exponential distribution based on upper record values. Math. Comput. Simul., 120, 64-78.

20. Dhanya, M. and Jeevavand, E. S. (2018). Stress-Strength Reliability of Power Function Distribution based on Records. J. Stat. Appl. Prob., 7(1), 39-48.

21. Dube, M., Krishna, H. and Garg, R. (2016). Generalized inverted exponential distribution under progressive first-failure censoring, J. Stat. Comput. Simul., 86(6), 1095-1114.

22. Efron, B. (1982). The Jackknife, the Bootstrap and Other Resembling Plans, SIAM, Philadelphia.

23. Ghitany, M.E., Tuan, V.K. and Balakrishnan, N. (2013). Likelihood estimation for a general class of inverse exponentiated distributions based on complete and progressively censored data. J. Stat. Comput. Simul., 84(1), 96-106.

24. Gunasekera, S. (2018). Classical, Bayesian and generalized inferences of the reliability of a multicomponent system with censored data. J. Stat. Comput. Simul., 88(18), 3455-3501.

25. Gupta, R. D. and Kundu, D. (1999). Generalized exponential distributions. Aust. N. Z. J. Stat., 41(2), 173-188.

26. Hassan, A. S., Abd-Allah, M. and Nagy, H.F. (2018). Estimation of $\mathrm{R}=\mathrm{P}(\mathrm{X}<\mathrm{Y})$ using record values from the generalized inverted exponential distribution. Pak. $\mathrm{j}$. stat. oper. res., 14(3), 645-660.

27. Hassan, A. S., Muhammed, H. Z. and Saad, M. S. (2015). Estimation of StressStrength Reliability for Exponentiated Inverted Weibull Distribution based on Lower Record Values. BJMCS, 11(2), 1-14.

28. Johnson, R. A. (1988). Stress-strength Models for Reliability, Handbook of Statistics (Vol. 7). Eds. P.R. Krishnaiah, and C.R. Rao. Amsterdam: NorthHolland.

29. Khan, M. J. S. and Arshad, M. (2016). UMVU Estimation of Reliability Function and Stress-Strength Reliability from Proportional Reversed Hazard Family based on Lower Records. Amer. J. Math. Management Sci., 35(2), 171-181.

30. Khan, M.J.S. and Khatoon, B. (2019). Statistical inferences of $\mathrm{R}=\mathrm{P}(\mathrm{X}<\mathrm{Y})$ for Exponential Distribution based on Generalized Order Statistics. Ann. Data Sci., DOI: https://doi.org/10.1007/s40745-019-00207-6.

31. Kotz, S., Lumelskii, Y. and Pensky, M. (2003). The Stress-strength Model and its Generalizations. Singapore: World Scientific.

32. Lehmann, E., L. and Casella, G. (1998). Theory of point estimation. SpringerVerlag, 2nd edition, NewYork. 
33. Mahmoud, M. A. W., EL-Sagheer, R. M., Soliman, A. A. and Abd-Ellah, A., H. (2016). Bayesian Estimation of $\mathrm{P}(\mathrm{Y}<\mathrm{X})$ based on Record Values from the Lomax Distribution and MCMC Technique. JMASM, 15(1), 488-510.

34. Panahi, H. (2017). Estimation Methods for the Generalized Inverted Exponential Distribution Under Type II Progressively Hybrid Censoring with Application to Spreading of Micro-Drops Data. Commun. Math. Stat., 5(2), 159-174.

35. Raqab, M. Z., Bdair, O. M. and Al-Aboud, F. M. (2018). Inference for the twoparameter bathtub-shaped distribution based on record data. Metrika, 81(3), 229253.

36. Rasethuntsa, T.R. and Nadar, M. (2018). Stress-strength reliability of a nonidentical-component-strengths system based on upper record values from the family of Kumaraswamy generalized distributions. Statistics, 52(3), 684-716.

37. Soliman, A. A., Abd-Ellah, A. H., Abou-Elheggag, N. A. and Ahmed, E. A. (2013). Reliability Estimation in Stress-Strength models: an MCMC approach. Statistics, 47(4), 715-728.

38. Tarvirdizade, B. and Ahmadpour, M. (2016). Estimation of the stress-strength reliability for the two-parameter bathtub-shaped lifetime distribution based on upper record values. Stat. Methodol., 31, 58-72.

39. Wasserman, L. (2003). All of statistics: a concise course in statistical inference. Springer, NewYork.

40. Wong, C. M. and Wu, Y. Y. (2009). A note on interval estimation of $\mathrm{P}(\mathrm{X}<\mathrm{Y})$ using lower record data from the generalized exponential distribution. Comput. Statist. Data Anal., 53(10), 3650-3658. 